\title{
Septicaemia and pleural effusion due to Plesiomonas shigelloides
}

\author{
.H. Humphreys ${ }^{1}$, B. Keogh ${ }^{2}$ and C.T. Keane ${ }^{1}$ \\ ${ }^{1}$ Department of Clinical Microbiology (Trinity College), St. James's Hospital, Dublin 8, and ${ }^{2}$ Meath Hospital, \\ Dublin 8, Republic of Ireland.
}

\begin{abstract}
Summary: Plesiomonas shigelloides is a rare cause of invasive infection, most clinically significant isolates being from the gastrointestinal tract of animals and man. Recently it has been implicated as an increasing cause of opportunistic infections.

We report a case of $\boldsymbol{P}$. shigelloides septicaemia and pleural effusion in a patient with pre-existing alcoholic liver disease. This case serves to illustrate the possible role of $P$. shigelloides as an opportunistic pathogen in a compromised host especially where there is co-existing liver disease.
\end{abstract}

\section{Introduction}

Plesiomonas shigelloides, a Gram negative facultative anaerobic bacterium, is a rare cause of invasive infection. Most clinically significant isolates have been recovered from the gastrointestinal tract of animals and man and it has been implicated as a cause of diarrhoea (Cooper \& Brown, 1968; Rolston \& Hopfer, 1984). It is being increasingly recognized, however, as an opportunist pathogen causing severe and lifethreatening illness. Meningitis plus septicaemia (Appelbaum et al., 1978), arthritis (Gordon et al., 1983), cholecystitis (Claesson et al., 1984) and endophthalmitis (Cohen et al., 1983) have all been recently described. Pleural effusion with septicaemia has not been previously reported; we describe such a case here.

\section{Case history}

A 64 year old man was admitted to the Meath Hospital, Dublin, in March 1985 complaining of right sided pleuritic chest pain sudden in onset, dyspnoea and a non-productive cough. In October 1984 he was investigated for a persistent right sided pleural effusion for which no cause was found. He was diagnosed, however, as having alcoholic liver disease.

On examination, he was tachypnoeic, had a pyrexia of $38.8^{\circ} \mathrm{C}$ and his blood pressure was $90 / 70 \mathrm{~mm} \mathrm{Hg}$. He had signs of a right pleural effusion and hepatomegaly.

A pleural tap was performed and a litre of straw coloured fluid was aspirated. Because of the seriousness of his condition, he was transferred to the

Correspondence: H. Humphreys, M.B., M.R.C.P.I.

Accepted: 12 December 1985
Intensive Care Unit and commenced on cefotaxime, two grams eight hourly, administered intravenously. In spite of the above measures and repeated pleural aspirations, the patient died 13 days after admission. Permission for a post-mortem examination was refused.

\section{Investigations}

Both blood cultures and pleural fluid taken on admission grew a Gram negative bacillus which was sensitive to tobramycin, cefotaxime and piperacillin. It was resistant to ampicillin and gentamicin. The Gram negative bacillus was later identified as Plesiomonas shigelloides using the API 20E system. The same organism was isolated from a repeat aspiration 3 days later.

Other investigations revealed an initially normal white cell count with a neutrophil leucocytosis later. The liver function tests were abnormal with a low serum albumin and prolonged prothrombin time. The pleural fluid contained total protein $25 \mathrm{~g} / 1$ and polymorphonuclear neutrophil leucocytes but no malignant cells.

\section{Discussion}

Plesiomonas shigelloides is a facultative Gram negative anaerobe formerly known as Aeromonas shigelloides. Its positive oxidase reaction distinguishes it from other Gram negative bacilli such as Escherichia coli and Serratia, while a positive indole reaction excludes it 
from the genus Pseudomonas. Unlike Aeromonas hydrophilia, it does not ferment mannitol (Topley \& Wilson, 1983).

Extra-intestinal infections due to this organism are increasingly being recognized and in many instances, there is a predisposing cause. Previous outbreaks of septicaemia (Appelbaum et al., 1970; Gordon et al., 1983; Ellner \& McCarthy, 1973) have occurred in the context of diverse clinical backgrounds including rheumatoid arthritis and sickle cell anaemia. Underlying liver disease was common to both this case and those reported by Gordon et al. (1983) and Ellner \& McCarthy (1973). Apart from the latter case, all died directly as a result of septicaemia.

To our knowledge this is the first reported case of $P$. shigelloides pleural effusion with septicaemia. The

\section{References}

APPELBAUM, P.C., BOWEN, A.J., ADHIKARI, M., ROBINSBROWNE, R.M. \& KOORNHOF, H.G. (1978). Neonatal septicaemia and meningitis due to Aeromonas shigelloides. Journal of Pediatrics, 92, 676.

CLAESSON, B.E.B., HOLMLUND, D.E.W., LINDHAGEN, C.A. \& MATZSCH, T.W. (1984). Plesiomonas shigelloides in acute cholecystitis: a case report. Journal of Clinical Microbiology, 20, 985.

COHEN, K.L., HOLYK, P.R., MCCARTHY, L.R. \& PEIFFER, L.R. (1983). Aeromonas hydrophilia and Plesiomonas shigelloides endophthalmitis. American Journal of Ophthalmology, 96, 403.

COOPER, R.G. \& BROWN, G.W. (1968). Plesiomonas shigelloides in South Australia. Journal of Clinical Pathology, 21, 715. source of the organism is unknown but most likely originated from the gastrointestinal tract. Colonic bacteria may bypass the filtering mechanism of the liver when there is associated liver disease as may have occurred in the case reported by Gordon et al. (1983), where $P$. shigelloides was isolated from the faeces on post-mortem. Despite eradication of the organism from both the blood and pleural fluid, infection due to this organism was largely responsible for the eventual outcome.

This case illustrates the importance of $P$. shigelloides as an opportunist pathogen in patients with a predisposing condition and emphasizes the fact that this is an organism whose pathology is not solely confined to the gastrointestinal tract.

ELLNER, P.D. \& MCCARTHY, L.R. (1973). Aeromonas shigelloides bacteraemia: a case report. American Journal of Clinical Pathology, 59, 216.

GORDON, D.L., PHILPOT, C.R. \& McGUIRE, C. (1983). Plesiomonas shigelloides septic arthritis complicating rheumatoid arthritis. Australia and New Zealand Journal of Medicine, 13, 275.

ROLSTON, K.V.I. \& HOPFER, R.L. (1984). Diarrhoea due to Plesiomonas shigelloides in cancer patients. Journal of Clinical Microbiology, 20, 597.

TOPLEY, W.W.C. \& WILSON, G. (1983). In Principles o Bacteriology, Virology and Immunology, Volume 2, Seventh Edition. p. 145 and p. 147. E. Arnold 